\title{
Absence of Klein's paradox for massive spinless bosons coupled by a nonminimal vector interaction
}

Luis B. Castro*

UNESP - Campus de Guaratinguetá, DFQ, São Paulo, Brazil

E-mail: benito@feg.unesp.br

\section{Tatiana R. Cardoso}

UNESP - Campus de Guaratinguetá, DFQ, São Paulo, Brazil

E-mail: cardoso@feg. unesp.br

\section{Antonio S. de Castro}

UNESP - Campus de Guaratinguetá, DFQ, São Paulo, Brazil

E-mail: castro@pq. cnpq.br

\begin{abstract}
A few properties of the nonminimal vector interaction in the Duffin-Kemmer-Petiau theory in the scalar sector are revised. In particular, it is shown that the nonminimal vector interaction has been erroneously applied to the description of elastic meson-nucleus scatterings and that the space component of the nonminimal vector interaction plays a peremptory role for the confinement of bosons whereas its time component contributes to the leakage. Scattering in a square step potential is used to show that Klein's paradox does not manifest in the case of a nonminimal vector coupling.
\end{abstract}

5th International School on Field Theory and Gravitation, April 20 - 242009

Cuiabá city, Brazil

\footnotetext{
* Poster section.
} 


\section{Contents}

1. Introduction 2

2. The DKP equation and the nonminimal vector coupling 3

3. The nonminimal vector step potential 4

4. Conclusions $\quad 6$

\section{Introduction}

The first-order Duffin-Kemmer-Petiau (DKP) formalism [1]-[2] describes spin-0 and spin-1 particles and has been used to analyze relativistic interactions of spin- 0 and spin- 1 hadrons with nuclei as an alternative to their conventional second-order Klein-Gordon and Proca counterparts. The onus of equivalence between the formalisms represented an objection to the DKP theory for a long time and only recently it was shown that they yield the same results in the case of minimally coupled vector interactions, on the condition that one correctly interprets the components of the DKP spinor [3]. The DKP formalism furnishes different second-order equations if scalar interactions are considered and enjoys a richness of couplings not capable of being expressed in the Klein-Gordon and Proca theories. A number of different couplings in the DKP formalism, with scalar and vector couplings in analogy with the Dirac phenomenology for proton-nucleus scattering, has been employed in the phenomenological treatment of the elastic meson-nucleus scattering at medium energies with a better agreement to the experimental data when compared to the Klein-Gordon and Proca based formalisms [4]-[9].

The purpose of the present Letter is to investigate some properties of the DKP theory in the scalar sector with the nonminimal vector coupling. Three-dimensional nonminimal vector potentials, added by other kinds of Lorentz structures, have already been used successfully in a phenomenological context for describing the scattering of mesons by nuclei [4]-[5], [7], [9]; by using one-dimensional potentials, it is shown that the nonminimal vector coupling has been improperly used in the phenomenology. It is pointed out that nonminimal vector potentials have some special features not displayed by minimal vector potentials. Scattering in a square step potential is used to show that Klein's paradox does not show its face in the case of a nonminimal potential, contrary to what occurs for a minimally coupled potential [10]. Furthermore, it is also shown that if the space component of the nonminimal potential exceeds its time component there will be a critical value for the potential strength which segregates two different possibilities for the waves beyond the potential interface, either a progressive wave or an evanescent wave, a circumstance that resembles the nonrelativistic result. If the space component of the nonminimal potential does not exceed its time component, though, the transmission coefficient will never vanish, in sharp contrast to the nonrelativistic quantum mechanics. 


\section{The DKP equation and the nonminimal vector coupling}

The DKP equation for a boson with interaction is given by [2] (with units in which $\hbar=c=1$ )

$$
\left(i \beta^{\mu} \partial_{\mu}-m-\mathbb{V}\right) \psi=0
$$

where the matrices $\beta^{\mu}$ satisfy the algebra

$$
\beta^{\mu} \beta^{v} \beta^{\lambda}+\beta^{\lambda} \beta^{v} \beta^{\mu}=g^{\mu v} \beta^{\lambda}+g^{\lambda v} \beta^{\mu}
$$

and the metric tensor is $g^{\mu v}=\operatorname{diag}(1,-1,-1,-1)$. The algebra expressed by (2.2) generates a set of 126 independent matrices whose irreducible representations are a trivial representation, a fivedimensional representation describing the spin-0 particles and a ten-dimensional representation associated to spin-1 particles. The DKP spinor has an excess of components and the theory has to be supplemented by an equation which allows to eliminate the redundant components. That constraint equation is obtained by multiplying the DKP equation by $1-\beta^{0} \beta^{0}$, namely

$$
i \beta^{k} \beta^{0} \beta^{0} \partial_{k} \psi=m\left(1-\beta^{0} \beta^{0}\right) \psi, \quad \mathrm{k} \text { runs from } 1 \text { to } 3
$$

The four-current $J^{\mu}=\bar{\psi} \beta^{\mu} \psi$ satisfies the equation

$$
\partial_{\mu} J^{\mu}-i \bar{\psi}\left(\mathbb{V}-\eta^{0} \mathbb{V}^{\dagger} \eta^{0}\right) \psi=0
$$

The time component of that current is not positive definite but it may be interpreted as a charge density. The adjoint spinor $\bar{\psi}=\psi^{\dagger} \eta^{0}$, with $\eta^{0}=2 \beta^{0} \beta^{0}-1$ in such a way that $\left(\eta^{0} \beta^{\mu}\right)^{\dagger}=\eta^{0} \beta^{\mu}$ (the matrices $\beta^{\mu}$ are Hermitian with respect to $\eta^{0}$ ). We can see from (2.4) that, if $\mathbb{V}$ is Hermitian with respect to $\eta^{0}$ then the four-current will be conserved. For the spin- 0 sector there are two scalar, two vector and two tensor terms [11]. By considering only the nonminimal vector terms, $\mathbb{V}$ is in the form

$$
\mathbb{V}=i\left[P, \beta^{\mu}\right] A_{\mu}
$$

where $P$ is a projection operator $\left(P^{2}=P\right.$ and $\left.P^{\dagger}=P\right)$ in such a way that $\bar{\psi} P \psi$ behaves as a scalar and $\bar{\psi}\left[P, \beta^{\mu}\right] \psi$ behaves like a vector. At this point it is also worthwhile to note that this matrix potential leads to a conserved four-current but the same does not happen if, instead of $i\left[P, \beta^{\mu}\right]$, one uses either $P \beta^{\mu}$ or $\beta^{\mu} P$, as in [4]-[5], [7], [9]. For the case of spin 0, we use the representation for the $\beta^{\mu}$ matrices given by [12]. Here the projection operator can be written as [11]

$$
P=\frac{1}{3}\left(\beta^{\mu} \beta_{\mu}-1\right)=\operatorname{diag}(1,0,0,0,0)
$$

In this case $P$ picks out the first component of the DKP spinor. If the terms in the potential $\mathbb{V}$ are time-independent, one can write $\psi(x, t)=\varphi(x) \exp (-i E t)$, where $\varphi^{T}=\left(\varphi_{1}, \ldots, \varphi_{5}\right)$, in such a way that the time-independent DKP equation for a scalar boson constrained to move along the $X$-axis decomposes into

$$
\begin{gathered}
\left(\frac{d^{2}}{d x^{2}}+\kappa^{2}\right) \varphi_{1}=0 \\
\varphi_{2}=\frac{E+i A_{0}}{m} \varphi_{1}, \quad \varphi_{3}=\frac{i}{m}\left(\frac{d}{d x}+A_{1}\right) \varphi_{1}, \quad \varphi_{4}=\varphi_{5}=0
\end{gathered}
$$


where

$$
\kappa^{2}=E^{2}-m^{2}+A_{0}^{2}-A_{1}^{2}+\frac{d A_{1}}{d x}
$$

For this time-independent problem, $J^{\mu}$ has the components

$$
J^{0}=2 \frac{E}{m}\left|\varphi_{1}\right|^{2}, \quad J^{1}=2 \frac{\operatorname{Im}\left(\frac{d \varphi_{1}}{d x} \varphi_{1}^{*}\right)}{m}, \quad J^{2}=J^{3}=0
$$

Since $J^{\mu}$ is not time dependent, $\varphi_{1}$ describes a stationary state. Note that only the first component of the spinor satisfies a Klein-Gordon-like equation, so that $\varphi_{1}$ and its first derivative are continuous even the potential suffers finite discontinuities. In this case of a discontinuous potential, $\varphi_{2}$ and $\varphi_{3}$ are discontinuous, but the discontinuity does not matter, because $J^{0}$ and $J^{1}$ only depend of $\varphi_{1}$ and its first derivative.

\section{The nonminimal vector step potential}

The one-dimensional square step potential is expressed as

$$
A_{\mu}=\theta(x) c_{\mu} V
$$

where $c_{\mu}$ are dimensionless and positive coupling constants constrained by $c_{0}+c_{1}=1, \theta(x)$ denotes the Heaviside step function and $V>0$ is the height of the step. For $x<0$ the DKP equation has the solution

$$
\varphi(x)=\varphi_{+} e^{+i k x}+\varphi_{-} e^{-i k x}
$$

where

$$
\varphi_{ \pm}^{T}=\frac{a_{ \pm}}{\sqrt{2}}\left(1, \frac{E}{m}, \mp \frac{k}{m}, 0,0\right)
$$

and $k=\sqrt{E^{2}-m^{2}}$. For $|E|>m$, the solution expressed by (3.2) and (3.3) describes plane waves propagating on both directions of the $x$-axis with the group velocity $v_{g}=d E / d k$ equal to the classical velocity. If we choose particles inciding on the potential barrier $(E>m), \varphi_{+} \exp (+i k x)$ will describe incident particles $\left(v_{g}=+k / E>0\right)$, whereas $\varphi_{-} \exp (-i k x)$ will describe reflected particles $\left(v_{g}=-k / E<0\right)$. The flux related to the current $J^{\mu}$, corresponding to $\varphi$ given by (3.2), is expressed as

$$
J^{1}=\frac{k}{m}\left(\left|a_{+}\right|^{2}-\left|a_{-}\right|^{2}\right)
$$

Note that the relation $J^{1}=J^{0} v_{g}$ maintains for the incident and reflected waves, since

$$
J_{ \pm}^{0}=\frac{E}{m}\left|a_{ \pm}\right|^{2}
$$

On the other hand, for $x>0$ one should have $v_{g} \geq 0$ in such a way that the solution in this region of space describes an evanescent wave or a progressive wave running away from the potential interface. The general solution has the form

$$
\varphi_{\mathrm{t}}(x)=\left(\varphi_{\mathrm{t}}\right)_{+} e^{+i q x}+\left(\varphi_{\mathrm{t}}\right)_{-} e^{-i q x}
$$


where

$$
\left(\varphi_{\mathrm{t}}\right)_{ \pm}^{T}=\frac{b_{ \pm}}{\sqrt{2}}\left(1, \frac{E+i c_{0} V}{m}, \frac{\mp q+i c_{1} V}{m}, 0,0\right)
$$

and

$$
q=\sqrt{k^{2}+\left(c_{0}-c_{1}\right) V^{2}}
$$

Due to the twofold possibility of signs for the energy of a stationary state, the solution involving $b_{-}$ can not be ruled out a priori. As a matter of fact, this term may describe a progressive wave with negative energy and phase velocity $v_{p h}=|E| / q>0$ (see, e.g. [10]). In other words, the solution $\left(\varphi_{\mathrm{t}}\right)_{-} \exp (-i q x)$ with $q \in \mathbb{R}$ reveals a signature of Klein's paradox. One can readily envisage that two different classes of solutions can be segregated:

- Class A. With $c_{1}>c_{0}$ for $V<V^{c}$, where

$$
V^{c}=\sqrt{\frac{E^{2}-m^{2}}{c_{1}-c_{0}}}
$$

or with $c_{1} \leq c_{0}$ for all $V$, one has $q \in \mathbb{R}$, and the solution describing a plane wave propagating in the positive direction of the $x$-axis with the group velocity $v_{g}=q / E$ is possible only if $b_{-}=0$. In this case the components of the current are given by

$$
J^{0}=\frac{E}{m}\left|b_{+}\right|^{2}, J^{1}=\frac{q}{m}\left|b_{+}\right|^{2}
$$

- Class B. With $c_{1}>c_{0}$ for $V>V^{c}$ one has that $q= \pm i|q|$, and (3.6) with $b_{\mp}=0$ describes an evanescent wave. The solution satisfying the requirement of finiteness at infinity requires $b_{\mp}=0$. In this case

$$
J^{0}=\frac{E}{m} e^{-2|q| x}\left|b_{ \pm}\right|^{2}, J^{1}=0
$$

Incidentally, the solution involving $b_{-}$is identical to the solution involving $b_{+}$, so we consider $b_{-}=0$.

The demand for continuity of $\varphi_{1}$ and $d \varphi_{1} / d x$ at $x=0$ fixes the wave amplitudes in terms of the amplitude of the incident wave and thus the reflection $(R)$ and transmission $(T)$ coefficients become

$$
R=\left\{\begin{array}{cc}
\left(\frac{k-q}{k+q}\right)^{2} & \text { for the class A } \\
1 & \text { for the class B }
\end{array}, \quad T=\left\{\begin{array}{cc}
\frac{4 k q}{(k+q)^{2}} & \text { for the class A } \\
0 & \text { for the class B }
\end{array}\right.\right.
$$

For all the classes one has $R+T=1$ as should be expected for a conserved quantity. Note that the charge density in (3.10) and (3.11) is always a positive quantity and so is $J^{1}$ in (3.10). This means that the scattered waves describe particles and not antiparticles, then Klein's paradox never comes to scenario.

For $c_{1}>c_{0}$ the transmission coefficient vanishes for a potential strength $V$ greater than the cutoff potential $V^{c}$. In fact, the mixed step potential behaves effectively as a ascending step and a similar situation occurs in nonrelativistic quantum mechanics. 
As for $c_{1} \leq c_{0}$, however, there is no cutoff potential. This is a result that runs counter our conceptions drawn from the nonrelativistic quantum mechanics. For $c_{1}=c_{0}$ the half-and-half mixed step potential is transparent $(T=1$ for all $V)$, and for $c_{1}<c_{0}$ the mixed step presents a transmission coefficient that vanishes asymptotically as $V \rightarrow \infty$. Those strange facts occur because the space component of the step potential behaves as an ascending step whereas its time component behaves as a descending step. For $c_{1}=c_{0}$ effects due to the time and the space components cancel each other and the mixed step potential effectively behaves as a null potential. For $c_{1}<c_{0}$ the tendency to a descending step dominates so that the mixed step potential effectively behaves as a descending step. Note that the reflection and transmission coefficients are the same for a wave incident from the right as for a wave incident from the left.

\section{Conclusions}

We have shown minimal and nonminimal vector interactions in the scalar sector of the DuffinKemmer-Petiau theory behave quite diversely. In particular, the nonminimal vector interaction has no counterpart in the Klein-Gordon theory. The nonminimal vector interaction has the very same effect on both particles and antiparticles and so it might be useful for boson-confining models. Scattering in a square step potential clearly shows that Klein's paradox, present in the case of a minimal coupling [10], is absent in the case of a nonminimal coupling. When the space component of the nonminimal potential does not exceed its time component, the transmission coefficient is different from zero even if the height of the step potential is extremely high. That odd result has been endorsed by observing the behaviour of the effective potential.

\section{References}

[1] G. Petiau, Acad. R. Belg., A. Sci. Mém. Collect. 16 (1936) No. 2 ; N. Kemmer, Proc. R. Soc. A 166 (1938) 127 ; R.J. Duffin, Phys. Rev. 54 (1938) 1114.

[2] N. Kemmer, Proc. R. Soc. A 173 (1939) 91.

[3] M. Nowakowski, Phys. Lett. A 244 (1998) 329; J.T. Lunardi, et al., Phys. Lett. A 268 (2000) 165.

[4] B.C. Clark, et al., Phys. Rev. Lett. 55 (1985) 592.

[5] G. Kälbermann, Phys. Rev. C 34 (1986) 2240; R.E. Kozack, et al., Phys. Rev. C 37 (1988) 2898; R.E. Kozack, Phys. Rev. C 40 (1989) 2181.

[6] V.K. Mishra, et al., Phys. Rev. C 43 (1991) 801.

[7] L.J. Kurth, et al., Phys. Rev. C 50 (1994) 2624; R.C. Barret, Y. Nedjadi, Nucl. Phys. A 585 (1995) 311c; L.J. Kurth, et al., Nucl. Phys. A 585 (1995) 335c.

[8] S. Ait-Tahar, J.S. Al-Khalili, Y. Nedjadi, Nucl. Phys. A 589 (1995) 307.

[9] B.C. Clark, et al., Phys. Lett. B 427 (1998) 231.

[10] T.R. Cardoso, L.B. Castro, A.S. de Castro, Phys. Lett. A 372 (2008) 5964.

[11] See, e.g., R.F. Guertin, T.L. Wilson, Phys. Rev. D 15 (1977) 1518.

[12] Y. Nedjadi, R.C. Barret, J. Phys. G 19 (1993) 87. 